\title{
Development of a Geriatric Fear of Falling Questionnaire for Assessing the Fear of Falling of Thai Elders
}

\author{
Punpissa Sangpring, PhD Candidate ${ }^{1)}$, Mantana Vongsirinavarat, PhD ${ }^{1)}$, \\ Vimonwan Hiengkaew, $\mathrm{PhD}^{1)}$, Jaranit Kaewkungwal, $\mathrm{PhD}^{2)}$ \\ 1) Faculty of Physical Therapy, Mahidol University: 999 Phuttamonthon 4 Road, Salaya, \\ Phuttamonthon, Nakhonpathom 73170, Thailand.TEL:+66 2-4415450,Email: punpissa@gmail.com \\ 2) Faculty of Tropical Medicine, Mahidol University
}

\begin{abstract}
Purpose] The fear of falling is a common problem among older people and it can lead to activity restriction and risk of future falls. A tool to assess the fear of falling in Thailand was not available; thus, it was essential to develop an appropriate questionnaire that can determine the severity of the fear of falling. [Subjects] Five hundred elderly subjects aged 60 years old or over participated in this study. [Methods] This study created a new questionnaire called the Thai Geriatric Fear of Falling Questionnaire consisting of 34 items with a 6-point Likert scale separated into 3 major domains: 15 physical and functioning items, 8 in environmental items and 11 psychosocial items. Then the psychometric properties of the new questionnaire were assessed. [Results] The reliability results show the internal consistency $(\mathrm{r}=0.965)$ and test-retest reliability $(\mathrm{r}=0.874)$ were excellent. The validity results for content $(4$ expert agreements), convergent $(\mathrm{r}=-0.910)$, and discriminative $(\mathrm{r}=0.122)$ were satisfactory. The best cut-off score of 66 was also identified from the acceptable area under the curve, sensitivity, and specificity $(0.794,90.1 \%$, and $100 \%$, respectively). [Conclusion] The Thai Geriatric Fear of Falling Questionnaire can be used as an instrument to assess the fear of falling. Utilizing this tool, health personnel can become aware of and better able to manage the problems associated with activity restriction from the fear of falling.

Key words: Fear of falling questionnaire, Psychometric properties, Thai elders
\end{abstract}

(This article was submitted Nov. 14, 2011, and was accepted Dec. 3, 2011)

\section{INTRODUCTION}

Falls have been identified as a serious issue for elderly persons and are a leading cause of injury, disability, and death. In recent years, various studies concerned falls as a community health problems in elders confirming by studies related to physical damage as head injuries, fracture, joint dislocations, and other serious soft tissue injuries ${ }^{1-3}$. Even when no physical injury occurs, falls can also lead to psychological fear of falling creating many problems ${ }^{1-6}$. The fear of falling and its sequelae frequently lead to dependence followed by functional deficits and a greater risk of falling $7-9$. The physical deconditioning caused by fear of falling was reported as decreasing endurance, coordination, muscle strength, and flexibility of the body, and reducing health and physical function. Moreover, fear of falling can reduce quality of life in many ways such as by limiting social contact or leisure activities $\Phi$.

Fear of falling was commonly believed to be a consequence of falls, a result of psychological trauma 10 . However, recent studies have revealed fear of falling in the elderly who have not fallen and found relationships between physical, psychological, and functional changes, $11-13$. The authors of these studies suggested that fear of falling might become a more pervasive and serious problem than falls, and it is an issue deserving of further study.

The most common approach to assess fear of falling is to ask directly, "How afraid of falling are you?" Although this method is informative, it may underestimate the actual incidence and might not detect possible variation in levels of fear across situations. Thus, other methods with quantitative approaches to assess fear of falling have been developed. Most of the common tools are constructed based on the self-efficacy framework, such as the Fall Efficacy Scale (FES) by Tinetti in 1990, the Activities-specific Balance Confidence Scale (ABC) by Powell \& Myers in 1995, the Survey of Activities and fear of falling in the elderly (SAFE) by Lachmann et al. in 1998. Nevertheless, these well known questionnaires are not appropriate for Thai elderly subjects because they were constructed for Western people who have different lifestyles. For example, walking on an icy sidewalk found in the FES, is inappropriate for Thailand. Thus, a fear of falling questionnaire for Thai subjects is warranted. Therefore, the objective of this study was to develop a questionnaire for identifying fear of falling by Thai elderly subjects which can distinguish the sources of activity avoidance related to falls in Thai elders. 


\section{SUBJECTS AND METHODS}

\section{Subjects}

The subjects were healthy elderly persons, both males and females, aged 60 years or over. They were volunteers who had an active lifestyle, went out socially and were able to understand and follow verbal instructions. They did not have cognitive impairment (score of at least 23 out of 30 points on the Thai Mental State Examination (TMSE) and were able to read Thai. The procedure of this study was approved by the Ethical Committee on Human Rights Related to Human Experimentation, Mahidol University. Subjects were explained the purpose and procedure of the study and asked to sign a consent form prior to participation.

\section{Methods}

In the questionnaire development process, the first step was to review the commonly used questionnaires for assessing fear of falling among the elderly, the FES, ABC, SAFE and other questionnaires that had been developed to assess fear of falling. These questionnaires were retrived from a literature review and discussion with healthcare providers who are interested in the geriatric field, especially the issue of falls. The items were pooled and grouped for inclusion in a draft of a new questionnaire which was tried out with a convenience group of elderly Thais. Open-ended questions were also asked about activities or situations that might cause falls to recruit other items relevant to Thai elderly.

After testing the draft, an appropriate format was selected and verified to match Thai elders. The meaning of each item was clarified and assessed as to whether they were appropriate. Four content experts in the geriatric field, especially in falls, who had experience of survey design reviewed the items for accuracy, wording, grammar, ambiguity, and other technical flaws. The types of answering scale were also determined and a 6-point Likert scale was chosen because it was easy to understand for elders and was also the best accurate scale to get the exactly answer comparing with other types. This draft was tried out in a preliminary study involving 100 subjects and exploratory factor analysis was also used to test the component factor of the developing questionnaire as well. The full version which called "The Thai Geriatric Fear of Falling Questionnaire" consists of 34 items with 3 major domains: 15 items in the physical and functioning domain, 8 items in the environmental domain, and 11 items in the psychosocial domain. The 6-point Likert scale ranging from 1 (not at all) to 6 (very much) was utilized for the answering system.

The psychometric properties of the questionnaire including validity and reliability were also tested for the overall concepts of the new questionnaire. The reliability testing compounded internal consistency and test-retest reliability. The retest session was done after 7 days. After that, the construct validity was tested determining by a combination of 2 aspects; the confirmatory factor analysis and the convergent and discriminative validity. In this study, convergent validity was established between the developing questionnaire and the previous questionnaire which was the Modified Thai Fall Efficacy Scale (MTFES) which consists of 14 items. This version of the MTFES was derived from 10 items of the original Fall Efficacy Scale (FES) developed by Tinetti et al. in 1990 and the last 4 items developed by Hill et al. in 1996 and was translated into Thai version by Chamonchant et al. in 2006 (see appendix A). The discriminative validity was tested with other questionnaires that might not evaluate psychological factors, especially fear, using the Thai Geriatric depression Scale (TGDS). The TGDS was developed by Train the Brain Forum Committee in 1994 with a Thai version composed of 30 items asking about both positive and negative feelings with a yes or no answering system. This questionnaire is used as a standard screening tool for assessing depression in elderly Thais ${ }^{16}$ (see appendix B). This is how the final version of the Thai Geriatric Fear of Falling Questionnaire was completed to assess the fear of falling of Thai elders.

For the psychometric properties statistical analysis, exploratory factor analysis was used to explore the component factors associated with fear of falling which were categorized into 3 major factors: physical and functioning, environmental, and psychosocial factors. The Cronbach's alpha and the Item to Total correlation were used to represent the internal consistency. The intraclass correlation coefficient (ICC3, 1) was used to assess test-retest reliability in this study. The Spearman Rho Sign Rank test was used to analyze the convergent and discriminative validities of the new questionnaire. Confirmatory factor analysis was also performed to confirm the 3 major factors of the questionnaire using Lisrel statistic.

Finally, the cut-off score to identify the degree of fear of falling of Thai elders was determined. The first question asking about fear of falling, which was developed by Tinetti et al., "Are you afraid of falling?" was set to be the global question to compare with the new questionnaire. The Receiver Operator Characteristic (ROC) curve, sensitivity, and specificity were calculated to determine the best cut-off score for discriminating the degree of fear of falling of Thai elderly. The ROC curve was constructed by calculating the sensitivity (true positive rate) and 1-specificity (false positive rate) as the cut-off change score defining a clinically meaningful change. The area under the curve (AUC) was also calculated to check the best cut-off point. Therefore, the degree of fear of falling in this study was defined by 2 levels, high and low fear of falling.

\section{RESULTS}

Five hundred elderly subjects, 354 females and 146 males, participated in this study. Their average age was $65.92 \pm 3.78$, ranging from 60 to 82 years old. There was no significant difference of age between the genders. The average Thai Mini Mental State Exam (TMSE) score of the subjects were $27.32 \pm 1.59$, ranging from 24 to 30 which showed there was no cognitive deficit among the elders. About one-third of the subjects reported no history of fall and no fear of falling, and less than 35 percent of the subjects reported a fall history. The subject characteristics are shown 
Table 1. Characteristics results of the elderly subjects

\begin{tabular}{cc} 
Variable & Mean \pm SD or N $(\%)$ \\
\hline Age (years) & $65.9 \pm 3.8$ \\
Female & $66.6 \pm 4.0$ \\
Male & $66.5 \pm 3.8$ \\
TMSE (scores) & $27.3 \pm 1.6$ \\
Female & $26.9 \pm 2.2$ \\
Male & $26.9 \pm 2.3$ \\
Gender & \\
Female & $354(70.8)$ \\
Male & $146(29.2)$ \\
Marital Status & \\
Married & $327(65.4)$ \\
Single & $173(34.6)$ \\
Living Status & \\
Alone & $43(8.6)$ \\
With Spouse & $84(16.8)$ \\
With Family & $373(74.6)$ \\
History of Falls & \\
No Falls & $325(65.0)$ \\
One Fall & $134(26.8)$ \\
Two or More Falls & $41(8.2)$ \\
Fear of Falling Reported & \\
No Fear & $332(66.4)$ \\
Fear & $168(33.6)$ \\
\hline
\end{tabular}

in Table 1.

The psychometric property results of the developing questionnaire were divided into 2 major properties: the validity and the reliability.

Content validity of the developing questionnaire was examined by 4 content experts, and all items were summarized and grouped according to the expert opinions into 3 major factors, physical and functioning, environmental, and psychosocial, before creating the final draft of the developing questionnaire. Confirmatory factor analysis also confirmed the 3 major domains of the new questionnaire.

The convergent validity was tested using the Modified Thai Fall Efficacy Scale (MTFES) ${ }^{15}$ and the discriminative validity was tested using the Thai Geriatric Depression Scale (TGDS) ${ }^{16}$ and both showed satisfactory results (Table 2).

For reliability testing, we tested the internal consistency, and the test-retest reliability. Internal consistency represented by Cronbach's alpha statistic 0.965 . This result implies that all items of the new questionnaire were well correlated with each others. The test-retest reliability of total scores represented by the Intraclass Correlation Coefficient (ICC 3, 1) were excellent $(r=0.874, p \leq 0.01)$.

The best cut-off score of the geriatric fear of falling questionnaire was calculated using the Received Operating Curve (ROC) and the Area Under the Curve (AUC). The result showed that the best cut-off score of this questionnaire was 66 with the best AUC of 0.794. It indicates that the elders who responded to this questionnaire with total scores of 66 and above are quite afraid of falling.

Also, to confirm that the score at 66 is the best cut-off point of the questionnaire for discrimination between those who are presumed to have fear and those who do not,
Table 2. Convergent and Discriminative Validity of the new questionnaire

\begin{tabular}{lc}
\hline Psychometric Properties & $\begin{array}{c}\text { Correlation } \\
\text { Co-efficient }\end{array}$ \\
\hline Convergent Validity: & \\
Total Score \& MTFES & $-0.910^{*}$ \\
Domain1: Physical Function \& MTFES & $-0.950^{*}$ \\
Domain2: Environmental \& MTFES & $-0.906^{*}$ \\
Domain3: Psychosocial \& MTFES & $-0.812^{*}$ \\
\hline Discriminative Validity: & \\
Total Score \& TGDS & $0.122^{*}$ \\
Domain1: Physical Function \& TGDS & $0.136^{*}$ \\
Domain2: Environmental \& TGDS & 0.077 \\
Domain3: Psychosocial \& TGDS & 0.084 \\
\hline * , significant differences at p < 0.01 &
\end{tabular}

diagnostic property tests including sensitivity, specificity, positive predictive value (PPV), and negative predictive value (NPV) were performed. The results of the diagnostic testing in comparison with other cut-off points are shown in Table 3.

These results mean that a cut-off score of 66 in this questionnaire can correctly identify $90.1 \%$ of elders who have fear and show a $76.1 \%$ likelihood that those with higher scores have fear, whereas below this level, those without fear were $100 \%$ correctly identified when they were tested by this questionnaire.

\section{DISCUSSION}

This study presents the new questionnaire called the Thai Geriatric Fear of Falling Questionnaire which was developed to meet the need for an instrument that can assess fear of falling for Thais. The concept of this questionnaire was constructed not only based on physical activities, but also based on functional, environmental, and psychosocial domains which affect fear of falling of Thai elders.

The final version of the questionnaire consisted of 34 items in 3 major domains determined by exploratory factor analysis. There were 15 items in the physical and functioning domain, 8 items in environmental domain and 11 items in psychosocial domain which were also confirmed by confirmatory factor analysis. The final version of the questionnaire could be beneficial for use as a guideline for monitoring fear of falling of individuals according to the main concern of each component. All elderly participants could complete this questionnaire within 10 minutes. This study used the self-report technique for respondents living in an urban community throughout the testing session; therefore the psychometric properties reported might not be applicable for other elderly populations.

The total scores of this questionnaire showed both good reliability and validity for psychometric properties. The face and the content validity also demonstrate that the final draft is conceptually acceptable and it was determined by consensus among experts in the geriatric field.

In this study, the results of convergent validity between 
Table 3. The diagnostic testing results of various cut-off points for the new questionnaire

\begin{tabular}{cccccc}
\hline Cut-off at & AUC & Sensitivity & Specificity & PPV & NPV \\
\hline 63 & 0.651 & 83.3 & 100 & 100 & 66.7 \\
64 & 0.683 & 86.7 & 100 & 100 & 71.5 \\
65 & 0.690 & 88.0 & 100 & 100 & 74.6 \\
66 & 0.794 & 90.1 & 100 & 100 & 76.1 \\
67 & 0.656 & 84.2 & 100 & 100 & 68.9 \\
68 & 0.637 & 79.8 & 100 & 100 & 66.3 \\
\hline
\end{tabular}

the Modified Thai Fall Efficacy Scale (MTFES) and the total score of the developing questionnaire as tested by the Spearman Rho Sign Rank correlation was $-0.910(p<0.01)$. This implies that both scales measure the same construct. The minus mark indicates the opposite direction of these scales. The MTFES was modified from the original version of the Fall Efficacy Scale, developed by Tinetti in $1990^{17}$, which is commonly used for investigating the fear of falling of Western elders. The new questionnaire showed a good correlation with the MTFES even though it contains some different items accommodating the different lifestyle of Thai people. The analysis of each domain of the new questionnaire also showed good correlation among domains as well as with the global fear of falling questionnaire.

The first domain, the physical and functioning factor showed high correlation with the MTFES $(r=-0.950, p<0.01)$. This finding strongly conforms to the FES and other questionnaires that assess fear of falling because these previous questionnaires were created based on the concept of self-efficacy which was directly reflected to physical performance of human $5,17,1$. The second domain, the environmental factor was also highly correlated with MTFES $(\mathrm{r}=-0.906, \mathrm{p}<0.01)$. Thus, these 2 domains denote the fear of falling related to the self-efficacy concept in the previous questionnaire construction. The concept strongly believes that the fall-related self efficacy addresses the association of the increased risk of falling and the decline in ability to perform activities of daily living and other physical tasks $7,8,17-20$. Previous studies hypothesized that fear of falling and fall self-efficacy have direct effects on a variety of measures of health and functional ability of the elderly $9,12,18,19$. The items in those measures are frequently associated with inactivity, restricted physical and social activity, limitation in risky situations, and decline in physical functioning which are directly related to the first 2 domains of the new questionnaire. Therefore, our findings confirm that the first 2 domains are highly correlated with previous fear of falling measures as demonstrated by the convergent validity.

The exploration of the psychosocial domain and the global MTFES questionnaire showed a correlation $(\mathrm{r}=-0.812$, $\mathrm{p}<0.01)$ similar to the first two domains. Nevertheless, this finding appears to be different from the hypothesis setting since the global questionnaire (MTFES) does not have any items related to psychological or social problems. Previous questionnaires have not emphasized the psychosocial domain because they are largely based on the self-efficacy concept related to fall. One possible reason for this result might be the indirect effect of fall injury which might cause mental problems. For example, the elderly might be aware of or faced with health care costs after falls or they might believe that they have some physical problems that might lead to falls. They also reported psychophysical characteristics such as heart palpitation, sweating, mood changes and hesitation. These results were confirmed by the results of the in-depth interview in the qualitative study. Our findings are consistent with the work of Huang et al. in 2006 who constructed the Fear of Falling Questionnaire in Taiwan. They reported that elderly people had psychosocial awareness especially of

life after falls. However, these findings differ from results of Western questionnaires, probably because of differences in lifestyle and culture between Western and Eastern countries, such as type of care received from family, confidence level, health status, etc ${ }^{8-10,21,22}$.

The other type of construct, the discriminative validity was tested by comparing the new questionnaire with the Thai Geriatric Depression Scale (TGDS). The correlation co-efficient of the total score of the new questionnaire and TGDS was $0.122(\mathrm{p}<0.01)$. This demonstrates that the construct of the new questionnaire does not measure depression phenomena. Although the new questionnaire has items that might interface with the psychological scale in the TGDS, these items are rather general compared with the items in the TGDS.

For further confirmation of the discriminative properties of the construct validity, the Spearman Rho Sign Rank test was used to analyze the correlations between the TGDS and each domain of the new questionnaire. The correlation co-efficient of the physical and functioning factor was $0.136(p<0.01)$ implying that both measures have different constructs. This is due to the physical and functioning domain recording the physical status of the elderly whereas the TGDS strongly describes the depressive feelings of the elderly. The investigation of the second domain, the environmental factor, also showed no correlation with the TGDS $(r=0.077)$, implying that items of this factor do not determine the same concept as the TGDS. However, it might be necessary to further investigate this domain, such as by performing Rasch Analysis, to eliminate some items which might be excluded from domain. Such research might improve on the fear of falling questionnaire, revealing a new short form of the questionnaire that could reduce testing time, or it may reveal levels of the severity of fear of falling in elders.

Surprisingly, there was also a low correlation between the psychosocial factor and the TGDS with no significance $(r=0.084, p=0.062)$. A possible explanation for this low correlation co-efficient might be the different scoring systems of the new questionnaire and the TGDS. The scoring system of the TGDS is yes or no while the scores of the new questionnaire range from 1 to 6 . This might affect the severity of the response to each item. Moreover, asking elderly Thais about psychosocial phenomenon might not elicit a direct answer, especially in the TGDS, as there are some items which would cause distress and discomfort.

The Thai Geriatric Fear of Falling Questionnaire had acceptable convergent and discriminative validity confirming the entire concept of construct validity.

The internal consistency of the questionnaire showed 
high inter-correlation with a Cronbach's alpha of 0.965 . Furthermore, additional analysis with item to total correlation that eliminating each item still showed high correlation among the remaining of them, confirming that there are no different aspects of fear of falling among items in the questionnaire. This finding indicates that although it includes psychological and social factors, the the new questionnaire still assesses the fear of falling of elderly Thais. Since the Thai Geriatric Fear of Falling Questionnaire was constructed based on the concept of a self-reported survey design, the reliability of the scale is of concern. In this study, the co-efficient of stability was 0.874 as represented by the Intraclass Correlation Coefficient (ICC 3,1), which demonstrates the excellent test-retest reliability of the questionnaire $(\mathrm{p} \leq 0.01)$. This should encourage other researchers to use it confidently as a screening test for elderly people with problems related to fear of falling.

The purpose of a cut-off score for the new questionnaire is to create the best criteria for discriminating elderly people who are presumed to have fear and those who do not by calculating sensitivity and specificity. The cut-off score is derived from the Received Operating Characteristic Curve (ROC) and is confirmed by the Area under the Curve (AUC). The results of this study indicate the score of 66 was the best cut-off score and 0.794 was the best AUC that could discriminate between elderly Thais with and without fear of falling. Most previous questionnaires do not have a cut-off score, except the FES which has a score of 70 as the cut-off point. However the original FES might not be appropriate for use with Thai people. Therefore, the cut-off score of the questionnaire investigated in this study may be of benefit for programs such as fall prevention programs for Thai elderly people. Furthermore, the sensitivity, specificity, positive predictive value, and negative predictive value were calculated to further explain the cut-off score. The score 66 could categorize $90.1 \%$ of elders tested by this questionnaire who had fear of falling (the sensitivity). It indicates that there was $76.1 \%$ chance that the elderly participants had fear of falling when they got a high score. Whereas the elders without fear of falling had low scores were $100 \%$ correctly indentify by this questionnaire. Thus, the score of 66 is acceptable to set as the cut-off score for the new Geriatric Fear of Falling Questionnaire to differentiate between Thai elderly people who have and do not have a fear of falling.

In summary, the psychometric property outcomes in the tool construction partially confirm that the Thai Geriatric Fear of Falling Questionnaire has excellent internal and testretest reliability which are at least as good as any existing measure of fear of falling. It also has good validity namely, face, content, convergent and discriminative validity confirming the whole conceptual construction for assessing fear of falling in the new questionnaire. The response to new questionnaire could be segregated into 3 major factors. These findings correlated to the 3 main concepts of the questionnaire which might be categorized as physical deficit, environmental hazards, and psychological problems. The results of this work have inspired us to study in greater detail the forms of belief, for example by gathering further in-depth qualitative information on Thai elders.

The Thai Geriatric Fear of Falling Questionnaire is the first tool constructed which directly measures fear of falling of Thai community-dwelling elders. The information from this study may help healthcare providers to understand elders' responses to their fear. It may also be used to identify elders at high risk of emotional distress related to fear of falling which might cause other deteriorations and health problems.

\section{REFERENCES}

1) Tinetti ME: Factors associated with serious injury during falls by ambulatory nursing home residents. J Am Geriatr Soc, 1987, 35: 644-648. Medline

2) Tinetti ME, Doucette J, Claus E, et al.: Risk factors for serious injury during falls by older persons in the community. J Am Geriatr Soc, 1995, 43: 1214-1221. [Medline

3) Tinetti ME, Speechley M: Prevention of falls among the elderly. N Engl J Med, 1989, 320: 1055-1059. [Medline CrossRef

4) Martin FC, Hart D, Spector T, et al.: Fear of falling limiting activity in young-old women is associated with reduced functional mobility rather than psychological factors. Age Ageing, 2005, 34: 281-287. Medline CrossRef

5) Tinetti ME, Mendes de Leon C, Doucette J, et al.: Fear of falling and fallrelated efficacy in relationship to functioning among community-living elders. J Gerontol, 1994, 49: M140-M147. Medline

6) Vellas BJ, Wayne S, Romero L, et al.: Fear of falling and restriction of mobility in elderly fallers. Age Ageing, 1997, 26: 189-193. Medline CrossRef

7) Lachman M, Howland J, Tennstedt S, et al.: Fear of falling and activity restriction: the survey of activities and fear of falling in the elderly (SAFE). J Gerontol Psycho Sci, 1998, 53B: P43-50. [CrossRef

8) Legters K: Fear of falling. Phys Ther, 2002, 82: 264-272. Medline

9) Li F, Fisher K, Harmer P, et al.: Fear of falling in elderly persons: associated with falls, functional ability, and quality of life. J Gerontol Psycho Sci, 2003, 58: P283-290. CrossRef

10) Nadine G, Alastair J: Fear of falling in the elderly. Geria Aging, 2003, 60: $15-17$.

11) Kressig R, Wolf S, Sattin R, et al.: Association of demographic, functional, and behavioral characteristics with activity-related fear of falling among older adults transitional to frailty. JAGS, 2001, 49: 1456-1462. [CrossRef

12) Lim K, Taylor L: Factors associated with physical activity among older people - a population-based study. Prev Med, 2005, 40: 33-40. [Medline] CrossRef

13) van Haastregt J, Zijlstra R, Rossum E, et al.: Feasibility of a cognitive behavioral group intervention to reduce fear of falling and associated avoidance of activity in community-living older people: a process evaluation. BMC Health Serv Res, 2007, 7: 156. [Medline] [CrossRef

14) Train the Brain Forum Committee (Forum TTB): Thai Mental State Examination (TMSE). Siriraj Hosp Gaz, 1993, 45: 359-374.

15) Chamonchant $D$, Boonyong $S$, Gaogasigam $C$ : A study in fear falling and Berg Balance Test in Thai elderly population. Thai J Physcial Ther, 2006, 28: $67-76$

16) Train the Brain Forum Committee (Forum TTB): Thai Geriatric Depression Scale. Siriraj Hosp Gaz, 1994, 46: 1-9.

17) Tinetti M, Richman D, Powell L: Falls efficacy as a measure of fear of falling. J Gerontol Psycho Sci, 1990, 45: 239-243.

18) Fuzhong L, Mcauley E, Fisher K, et al.: Self-efficacy as a mediator between fear of falling and functional ability in the elderly. J Aging Health, 2002, 14: 452-466. Medline] [CrossRef

19) Li F, Fisher A, Harmer P, et al.: Falls self-efficacy as a mediator of fear of falling in an exercise intervention for older adults. J Gerontol. Psycho Sci, 2005, 60B: 34-40.

20) Powell LE, Myers A: The Activities-specific Balance Confidence (ABC) Scale. J Gerontol A Biol Sci Med Sci, 1995, 50A: M28-M34. Medline CrossRef

21) Huang TT: Geriatric fear of falling measure: development and psychometric testing. Int J Nurs Stud, 2006, 43: 357-365. [Medline [CrossRef]

22) Huang $\mathrm{HC}$, Gau M, Lin W, et al.: Assessing risk of falling on older adults. Public Health Nurs, 2003, 20: 399-411. Medline [CrossRef 


\section{Appendix}

Appendix A: Items of the Modified Thai Fall Efficacy Scale (MTFES) translated into Thai version by Chamonchant et al. in 2006

\begin{tabular}{|c|c|c|}
\hline Item & $\begin{array}{c}\text { Very much fear } \\
(0)\end{array}$ & $\begin{array}{c}\text { No fear } \\
(10)\end{array}$ \\
\hline \multicolumn{3}{|l|}{ 1. Get dressed and undressed } \\
\hline \multicolumn{3}{|l|}{ 2. Prepare meals } \\
\hline \multicolumn{3}{|l|}{ 3. Take a bath } \\
\hline \multicolumn{3}{|l|}{ 4. Get in or out of a chair } \\
\hline \multicolumn{3}{|l|}{ 5. Get in or out of bed } \\
\hline \multicolumn{3}{|l|}{ 6. Answer the door or telephone } \\
\hline \multicolumn{3}{|l|}{ 7. Walk around the house } \\
\hline \multicolumn{3}{|l|}{ 8. Reach into cabinets/closets } \\
\hline \multicolumn{3}{|l|}{ 9. Light housekeeping } \\
\hline \multicolumn{3}{|l|}{ 10. Simple shopping } \\
\hline \multicolumn{3}{|l|}{ 11. Use public transportation } \\
\hline \multicolumn{3}{|l|}{ 12. Cross the road } \\
\hline \multicolumn{3}{|l|}{ 13. Light gardening or hanging out the washing } \\
\hline 14. Using front or rear steps at home & & \\
\hline
\end{tabular}

Appendix B: The Thai Geriatric Depression Scale (TGDS) constructed by Train the Brain Forum Committee in 1994

\begin{tabular}{|c|c|c|}
\hline Choose the best answer for how you felt over the past week & Yes & No \\
\hline 1. Are you basically satisfied with your life? & & \\
\hline 2. Have you dropped many of your activities and interests? & & \\
\hline 3. Do you feel that your life is empty? & & \\
\hline 4. Do you often get bored? & & \\
\hline 5. Are you bothered by thoughts you can $t$ get out of your head? & & \\
\hline 6. Are you in good spirits most of the time? & & \\
\hline 7. Are you afraid that something bad is going to happen to you? & & \\
\hline 8. Do you feel happy most of the time? & & \\
\hline 9. Are you hopeful about the future? & & \\
\hline 10. Do you often feel helpless? & & \\
\hline 11. Do you often get restless and fidgety? & & \\
\hline 12. Do you prefer to stay at home, rather than going out and doing new things? & & \\
\hline 13. Do you frequently worry about the future? & & \\
\hline 14. Do you feel you have more problems with memory than most? & & \\
\hline 15. Do you think it is wonderful to be alive now? & & \\
\hline 16. Do you often feel downhearted and blue? & & \\
\hline 17. Do you feel pretty worthless the way you are now? & & \\
\hline 18. Do you worry a lot about the past? & & \\
\hline 19. Do you find life very exciting? & & \\
\hline 20. Is it hard for you to get started on new projects? & & \\
\hline 21. Do you feel full of energy? & & \\
\hline 22. Do you feel that your situation is hopeless? & & \\
\hline 23. Do you think that most people are better off than you are? & & \\
\hline 24. Do you frequently get upset over little things? & & \\
\hline 25. Do you frequently feel like crying? & & \\
\hline 26. Do you have trouble concentrating? & & \\
\hline 27. Do you enjoy getting up in the morning? & & \\
\hline 28. Do you prefer to avoid social gatherings? & & \\
\hline 29. Is it easy for you to make decisions? & & \\
\hline 30. Is your mind as clear as it used to be? & & \\
\hline
\end{tabular}

